\title{
LINEAR TOPOLOGICAL SPACES
}

\section{H. HYERS}

1. Introduction. In general analysis it is customary to study linear spaces for which there is defined a "norm," which takes the place of the absolute value of ordinary analysis in defining distance, limit point, continuity, and so on. Linear metric spaces more general than the normed spaces have also been studied by Fréchet, Banach, and several others. In line with the trend toward general topology, it seems natural to generalize still more by introducing linear topological spaces, that is, linear spaces which are at the same time topological spaces, in which the fundamental "linear" operations of addition and scalar multiplication are continuous. We shall always assume that the topology is subject to the axioms for a $T_{1}$-space. ${ }^{1}$

The topology of $T_{1}$-spaces may be introduced in various ways; by postulating a system of open sets or of neighborhoods with certain properties, and so on. We shall find it convenient to give a set of postulates for the topology of the linear space $L$ in which "neighborhood" is the fundamental undefined notion. Since $L$ is a topological group, it has a uniform topology and hence it is sufficient to consider neighborhoods of the origin. Moreover, the "uniform structure" implies that $L$ is a completely regular Hausdorff space. ${ }^{2}$

The following notations will be used. The set of elements $x$ having the property $P$ will be denoted by $\{x ; P\}$. If $S$ and $T$ are subsets of $L, \alpha$ is a fixed real number, and $x$ a fixed point of $L, x+S$ denotes the set $\{x+y ; y \in S\} ; S+T$ denotes the set $\{y+z ; y \in S, z \in T\} ; \alpha S$ stands for the set $\{\alpha y ; y \in S\}$. The notations $\cup$ and $\cap$ are used for union and intersection, respectively. The following definition of a linear topological space is equivalent to the one given above. ${ }^{3}$

Definition 1.1. A linear space $L$ will be called a linear topological space (abbreviated 1.t.s.) if and only if there is a system $V$ of subsets

An address delivered before the April meeting of the Society in Berkeley on April 29, 1944, by invitation of the Program Committee; received by the editors July 17, 1944.

1 The definition given here seems to be due to Kolmogoroff [1], 1934. However, a linear space with a more general topology than that of a $T_{1}$-space was defined by Frechet $[2,3]$ in 1926 under the name "topological affine space." The postulates for a $T_{1}$-space are given in Alexandroff and Hopf $[1$, p. 59]. The bracketed numbers refer to the bibliography.

2 A. Weil $[1$, p. 13].

${ }^{3}$ For the proof see Hyers [4]. 
$U$ of $L$ called neighborhoods of the origin subject to the following postulates. ${ }^{4}$

(i) The intersection $\cap\{U ; U \in U\}$ of all the members of $U$ is the one element set consisting of the origin $\theta$.

(ii) Given $U \in V, V \in V$, there exists $W \in \mathcal{V}$ such that $W \subset U \cap V$.

(iii) If $U \in U$ there exists $V \in U$ such that $V+V \subset U$.

(iv) Given $U \in \mathcal{U}$ there exists $V \in \mathcal{U}$ such that $\alpha V \subset U$, for all real $\alpha$ satisfying $-1 \leqq \alpha \leqq 1$.

(v) For every $x \in L$ and $U \in V$, there exists a real number $\alpha$ such that $x \in \alpha U$.

Linear topological spaces may be regarded on the one hand as very special topological groups, which are abelian and have real "operators," or on the other hand as a generalization of Fréchet spaces, Banach spaces, and so on.

We now consider some examples of 1.t.s.

EXAMPLE 1. The space of all real functions $x(t)$ of a real variable $t$ on a fixed interval (the interval may be finite or infinite). The neighborhoods $U$ of the origin are taken to be the sets $U(\sigma, \delta)=\{x ;|x(t)|<\delta$, $t \in \sigma\}$, where $\sigma$ ranges over all finite sets of real numbers on the interval, and $\delta$ ranges over all positive numbers.

EXAMPLE 2a. Any linear normed space $E$ with its usual "strong" topology. Put $U(\delta)=\{x ;\|x\|<\delta\}$, where $\delta>0$.

EXAMPLE 2b. ${ }^{5} A$ linear normed space $E$ with its weak topology. Corresponding to each finite set $\sigma$ of linear continuous functionals $f(x)$ on $E$, and each $\delta>0$, define $U(\sigma, \delta)=\{x ;|f(x)|<\delta, f \in \sigma\}$. A linear normed space $E$ with its weak topology, considered as a l.t.s., will be denoted by $\varepsilon$.

EXAMPLE 3. The space $l^{1 / 2}$ of sequences $x=\left(x_{1}, x_{2}, \cdots\right)$ such that $|x|=\left(\sum_{i=1}^{\infty}\left|x_{i}\right|^{1 / 2}\right)^{2}<\infty$. The neighborhoods $U$ are defined to be the sets $U(\delta)=\{x ;|x|<\delta\}$, where $\delta>0$.

The space $\varepsilon$ of Example $2 \mathrm{~b}$ is of considerable importance in some recent developments of Banach space theory. In Example 1 the topology is simply that of "point-wise" convergence. This example illustrates the utility of the concept of a l.t.s. in situations where pointwise convergence is desired. The norm or "strong" topologies on the other hand (Example 2a) apply to function spaces in which the convergence of elements means the uniform convergence of functions, or convergence in the mean, and so on.

Example 1 may be generalized by considering the topological prod-

${ }^{4}$ Except for (i), these postulates are identical to those given by von Neumann [1].

5 For further examples of a similar nature related to Hilbert space see von Neumann $[1,2]$. 
uct of a family of 1.t.s. Let $A$ be any abstract set of arbitrary cardinal number, and let there be assigned to each $a \in A$, a l.t.s. $L_{a}$. The space $\Pi L_{a}$ of functions $x(a)$, with $x(a) \in L_{a}$ for each $a \in A$, will be called the linear topological product of the family $\left\{L_{a} ; a \in A\right\}$ of 1.t.s. when addition and scalar multiplication are defined in the usual manner, and a typical neighborhood $U$ of the origin in $\Pi L_{a}$ is defined as

$$
U=\left\{x(a) ; x\left(a_{i}\right) \in U_{a_{i}}, i=1, \cdots, n\right\},
$$

where $a_{1}, \cdots, a_{n}$ is any finite set of indices and $U_{a}$ is any neighborhood of the origin in the space $L_{a}$. It is easily shown that the linear topological product of a family of 1.t.s. is itself a 1.t.s. According to this definition, Example 1 may be thought of as the space of real numbers raised to the power of the continuum. The linear topological product of a finite number of normed spaces is itself a normed space, but this is not true for an infinite family of normed spaces. ${ }^{6}$

2. The properties of boundedness and convexity; normability and metrizability. One of the first questions to come up in studying 1.t.s. is to determine conditions under which the space under consideration is capable of being normed or metrized, and hence subsumed under more traditional systems.

We shall call two 1.t.s. isomorphic if they are isomorphic as abstract linear spaces and if in addition the isomorphism is bicontinuous. A necessary and sufficient condition for a l.t.s. to be normable, that is, isomorphic to a normed space, was given by Kolmogoroff [1] in terms of two geometrical properties which will now be defined. The term "convex set" will be used in its usual sense: $S$ is convex if $x$, $y \in S, 0<\alpha<1$ imply $\alpha x+(1-\alpha) y \in S$. A subset $S$ of a 1.t.s. $L$ will be said to be bounded if for any neighborhood $U$ of the origin there is a real number $\alpha$ such that $S \subset \alpha U$. This definition was given by von Neumann [1]. It can be shown ${ }^{7}$ to be equivalent to an earlier definition due to Banach. Another equivalent definition of boundedness was given by Michal and Paxson [1].

THEOREM 1 (Kolmogoroff [1]). A necessary and sufficient condition for a l.t.s. to be normable is that it contain an open set which is both convex and bounded.

The central idea of the proof is the Minkowski functional, which is used to define the norm:

$$
\|x\|=\inf \{\alpha ; x \in \alpha U\},
$$

6 See for example Bourgin [1, p. 639].

' Hyers [3]. 
where $U$ is a bounded, open, symmetric, convex neighborhood of the origin. The triangular property of the norm is a consequence of the convexity of the fundamental neighborhood $U$. The property that $\|x\|=0$ implies $x=\theta$ follows from the fact that since $U$ is bounded, the neighborhoods $U^{\prime}=(1 / n) U, n=1,2,3, \cdots$, form a complete neighborhood system of the origin.

It has been pointed out ${ }^{8}$ that the next theorem can be easily deduced from a theorem of G. Birkhoff [1] on the metrization of topological groups.

Theorem 2. A l.t.s. $L$ is metrizable as a space of type $F$ (not necessarily complete) if and only if there exists a countable complete neighborhood system of the origin in $L$.

The following definitions are suggested by the results of Theorem 1 .

Definition 2.1. A 1.t.s. $L$ is called locally convex ${ }^{9}$ if there exists a complete neighborhood system of the origin, every neighborhood of which is convex.

Definition 2.2. A 1.t.s. $L$ is called locally bounded if it contains a non-void bounded open set (Hyers [4]).

To return for a moment to the examples given in $\$ 1$, we see that Examples $1,2 \mathrm{a}$ and $2 \mathrm{~b}$ are locally convex. The space $l^{1 / 2}$ of Example 3 is locally bounded but not locally convex (Tychonoff [1]). Examples 1 and $2 \mathrm{~b}$ are, in general, spaces which do not have countable complete neighborhood systems of the origin and hence are not metrizable. This is easily demonstrated for Example 1. It was proved by von Neumann [2] for weak Hilbert space $\mathfrak{H C}$ by constructing a set $S \subset \mathfrak{H C}$ which had the origin as a limit point, and yet had the property that no sequence of the elements of $S$ was convergent to the origin. Thus in non-metrizable 1.t.s. the ordinary concept of sequential convergence may not be sufficient to give us all the limit points of the space. The space $\varepsilon$ of Example $2 \mathrm{~b}$ is normable if and only if it is finite-dimensional (Wehausen [1]).

The property of local convexity plays an important role in many analytical theories, especially in the theories of linear functionals, integrals, and functional equations. Fortunately, most of the more important instances of 1.t.s. do have the property of local convexity. It is easily seen that the linear topological product of an arbitrary family of locally convex spaces is itself locally convex. ${ }^{10}$

The property of local boundedness ${ }^{11}$ puts a rather severe restriction

${ }^{8}$ Hyers [3], Wehausen [1].

-The term "convex" is used by von Neumann [1] in place of "locally convex."

${ }^{10}$ This was pointed out by Bourgin [1].

il Defined by Hyers [4]. 
on the 1.t.s., as the following theorems show. It was mentioned in connection with Theorem 1 that the property of local boundedness implies that there exists a countable complete neighborhood system of the origin. Thus from Theorem 2 we have:

THEOREM 3. Every locally bounded l.t.s. is metrizable.

Again, by carrying through the same type of argument used in proving Theorem 1 , one can prove $\mathrm{e}^{12}$ the following theorem (the functional $|x|$ is again defined by formula (1), but without requiring the convexity of $U$ ).

THEOREM 4. If $L$ is a locally bounded l.t.s. there exists a non-negative real valued functional $x$ on $L$ with the following properties:

(i) $|x|=0$ implies that $x=\theta$;

(ii) $|\alpha x|=|\alpha| \cdot|x|$ for every $x$ in $L$ and every real $\alpha$;

(iii) there exists a real number $\beta \geqq 1$ such that $|x+y| \leqq \beta(|x|+|y|)$ for all $x$ and $y$ in $L$;

(iv) the sets $S(\delta)=\{x ; x \in L,|x|<\delta\}$, where $\delta>0$, form a complete neighborhood system of the origin.

Conversely, if $L$ is a linear space and there exists a non-negative real valued functional $|x|$ defined on $L$ and having the properties (i), (ii), (iii), then $L$ is a locally bounded l.t.s., when the topology is defined in accordance with (iv).

The multiplier property (iii) is due to Bourgin [1]. The properties given by Hyers [4] were (i), (ii), (iv) and in place of (iii) an apparently weaker continuity property, that $|x+y| \rightarrow 0$ whenever $|x| \rightarrow 0$ and $|y| \rightarrow 0$. However, on the assumption of this continuity property and properties (i) and (ii) it is easily shown that (iii) holds. ${ }^{13}$ For if $|x| \leqq \delta,|y| \leqq \delta$ imply that $|x+y|<1$, then choose any $x_{1}$ and $y_{1}$ such that either $x_{1}$ or $y_{1}$ is not the zero element. Putting $x=\delta \cdot x_{1} /\left(\left|x_{1}\right|+\left|y_{1}\right|\right)$ and $y=\delta \cdot y_{1} /\left(\left|x_{1}\right|+\left|y_{1}\right|\right)$ gives $\left|x_{1}+y_{1}\right|$ $<\beta\left(\left|x_{1}\right|+\left|y_{1}\right|\right)$, where $\beta=1 / \delta$. On the other hand, if $\left|x_{1}\right|=\left|y_{1}\right|=0$, the inequality (iii) is obviously satisfied.

Definition 2.3. The functional $|x|$ appearing in Theorem 4 will be called a quasi-norm, and $\beta$ is called a multiplier of the quasi-norm.

One is tempted to look for the least multiplier for a given locally bounded space. However, Bourgin [1] has shown by an example that there may be no least multiplier. By Theorem 1 it is possible to take $\beta=1$ if and only if the space is locally convex.

In normed spaces the triangular inequality implies that the norm

12 For the proof see Hyers [3] and Bourgin [1].

${ }^{13}$ A different proof was given by Bourgin. 
is continuous at every point of the space. It is easily shown by an example that the properties (i), (ii), (iii), (iv) do not imply continuity everywhere. However, if the set $U$ is bounded and open, the quasinorm obtained by formula (1) is everywhere upper semi-continuous (Hyers [4]). The complete answer to this continuity question was recently given by Bourgin [1] who proved the following theorem.

THEOREM 5. In any locally bounded l.t.s. $L$ it is possible to define a quasi-norm with the properties (i), (ii), (iii), (iv) of Theorem 4, and with the additional property: (v) $|x|$ is continuous in $x$ at each point of $L$.

Bourgin's proof, of course, involves a suitable selection of the fundamental neighborhood $U$ occurring in the definition of the quasinorm (see formula (1)). This neighborhood was chosen to be a sphere $\{x ; \rho(x, \theta)<\alpha\}$, where $\rho(x, y)$ is a certain metric for the space (see Theorem 3). A particular case of a locally bounded 1.t.s. with is not normable is the space $l^{1 / 2}$ (Example 3). Here the quasi-norm can be defined as $|x|=\left(\sum_{i=1}^{\infty}\left|x_{i}\right|^{1 / 2}\right)^{2}$, and in this case the number 2 is a multiplier. Further examples are given by M. M. Day [1].

The following theorem shows that the results of requiring local compactness are the same for a 1.t.s. as for a linear normed space. The term compact will be used in the "old fashioned" sense, that is, a set $S$ is compact if every infinite subset of $S$ has a limit point in $S$.

THEOREM 6, A necessary and sufficient condition for a l.t.s. $L$ to be isomorphic to a finite-dimensional Euclidean space is that $L$ be locally compact, that is, that $L$ contain an open set whose closure is compact.

Theorem 6 may be proved by first showing that local compactness implies local boundedness, and hence the existence of a quasi-norm. The existence of a finite basis is then established by the method of F. Riesz [1], who proved the theorem for the space $C$ of continuous functions. ${ }^{14}$

This result suggests the possibility of extending the Riesz theory of completely continuous linear functional equations to transformations of 1.t.s. However, so far it has been found possible to do this only for locally bounded spaces (Hyers [4]).

3. Fixed point theorems and functional equations. The well known fixed point theorem of Brouwer for continuous transformations of an $n$-dimensional topological simplex into itself has been generalized to compact subsets of (possibly) infinitely-dimensional spaces by several

14 Theorem 6 may also be deduced from Theorem 43 of Pontrjagin [1, p. 170]. 
authors, ${ }^{15}$ beginning with Birkhoff and Kellogg [1]. The numerous applications of such fixed point theorems to the proof of existence theorems for both ordinary and partial differential equations as well as to other functional equations are too well known to require extensive reiteration here. The Brouwer fixed point theorem was generalized to the case of bicompact convex subsets of a locally convex 1.t.s. by Tychonoff [1], who also applied his results to obtain an existence theorem for infinite systems of differential equations. Tychonoff's theorem was also used by Paxson $[1,3]$ in the study of differential equations for functions whose values are in a 1.t.s.

As beautiful as the concepts and methods are in this general approach to the solution of functional equations, it does have certain disadvantages. The method is "non-effective" in the sense that no method is given for constructing a set of approximations to the fixed point. Moreover, the method leads only to the existence of a fixed point, and tells us nothing about its uniqueness.

Another and older method for proving existence theorems for functional equations is the famous method of successive approximations of Picard. This method has the advantage of being "effective," and hence capable of being applied to obtain approximate solutions. It also provides a uniqueness proof. The "heavy" restriction made here is that the transformation involved satisfies a Lipschitz condition. An application of Picard's 'method to the proof of a fixed-point theorem for transformations of a subset of a complete metric space was given by Hildebrandt and Graves [1]. ${ }^{16}$ The author's Theorem 7 below ${ }^{17}$ is a generalization of the result of Hildebrandt and Graves to transformations operating on 1.t.s. In order to formulate a suitable analog of the Lipschitz condition in a 1.t.s. we need the following definition.

Definition 3.1. By a Lipschitz system for a 1.t.s. $L$ we shall understand a family of sets $K(x)$, with the following properties:

(1) To each $x$ in $L$ there corresponds a convex set $K(x)$.

(2) $x \in K(x)$ and $\theta \in K(x)$; if $x \neq \theta$ and $\alpha>1$, then $\alpha x \in K(x)$.

(3) $K(\alpha x)=\alpha K(x)$ for $\alpha \geqq 0$.

(4) $y \in K(x)$ implies $K(y) \subset K(x)$.

(5) Each $K(x)$ is bounded.

${ }^{15}$ For example, Schauder [1]. Further references are given by Graves [1]. An application of a fixed point theorem to an existence theorem arising in a hydrodynamical problem was worked out by Weyl [1].

${ }^{16}$ For a discussion see Graves [1].

${ }^{17}$ Hyers $[1,2]$. 
In the special case of a linear normed space $E$, the sets $K(x)$ $=\{y ;\|y\| \leqq\|x\|\}$ form a Lipschitz system. A sequence $x_{n} \in L$ will be called fundamental if, corresponding to each neighborhood $U$ of the origin, there is a positive integer $n_{0}$ such that $x_{m}-x_{n} \in U$ for $m>n_{0}$ and $n>n_{0}$. A subset $S$ of $L$ will be called sequentially complete if every fundamental sequence $x_{n} \in S$ converges to a point of $S$. The closure of $S$, that is, the set consisting of $S$ and its limit points, is denoted by $\bar{S}$.

THEOREM 7. Let $z=f(y)$ be a continuous transformation of $Y$ into part of itself, where $Y$ is a sequentially complete subset of a linear topological space L. Let $\{K(y)\}$ be any Lipschitz system with respect to which the function $f(y)$ satisfies the Lipschitz condition

$$
f(y)-f(z) \in \mu K(y-z)
$$

for all pairs $y, z \in Y$, where $\mu$ is a positive constant less than one. Then the transformation $z=f(y)$ of $Y$ into part of itself has a unique fixed point.

PRoof. For any chosen $y_{0} \in Y$, define $y_{n}$ recurrently by $y_{n+1}=f\left(y_{n}\right)$, $n=0,1,2, \cdots$, so that $y_{n} \in Y$ for all $n$. Using properties (3) and (4) of Lipschitz systems and an induction we obtain

$$
y_{n+1}-y_{n} \in \mu^{n} K\left(y_{1}-y_{0}\right), \quad n=1,2, \cdots \text {. }
$$

Hence we have

$$
\begin{aligned}
y_{m+p}-y_{m} & =y_{m+p}-y_{m+p-1}+\cdots+y_{m+1}-y_{m} \\
& \in \mu^{m+p-1} K\left(y_{1}-y_{0}\right)+\cdots+\mu^{m} K\left(y_{1}-y_{0}\right) \\
& \in\left(\mu^{m+p-1}+\cdots+\mu^{m}\right) K\left(y_{1}-y_{0}\right),
\end{aligned}
$$

where the last step is justified since by (1), $K\left(y_{1}-y_{0}\right)$ is a convex set. By (5) the set $K\left(y_{1}-y_{0}\right)$ is bounded, so that there is a $\delta>0$ corresponding to each neighborhood $U$ of the origin $\theta$ such that $0<\beta<\delta$ implies $\beta K\left(y_{1}-y_{0}\right) \subset U$. Now $0<\mu<1$ so that $\sum \mu^{n}$ converges, and for sufficiently large $m$ and any $p$ we have $y_{m+p}-y_{m} \in U$, whence $y_{n}$ is a fundamental sequence. The existence of a fixed point now follows immediately, while the uniqueness is readily proved by using property (2) together with the inequality $0<\mu<1$.

TheOREM 8. In any locally convex linear topological space there always exist complete neighborhood systems $V:(U, V, W, \cdots)$ of the origin with the properties:

(i) if $U \in V$, then $U$ is convex;

(ii) if $U \in U$ then $\alpha U \in U$, for each real $\alpha$. 
For any such neighborhood system $U$ put $K(x)=\cap\{U ; U \in V, x \in U\}$. Then the sets $K(x)$ form a Lipschitz system for the space $L$ and we have $K(x)=\cap\{\bar{U} ; U \in V, x \in \bar{U}\}$.

This theorem gives us a useful way of finding Lipschitz systems in any particular locally convex space. For instance, in weakly topologized Hilbert space $\mathfrak{T C}$ the theorem tells us that the sets $K(x)$ of all $z$ for which $|(z, y)| \leqq|(x, y)|$, as $y$ runs over $\mathfrak{F}$, form a Lipschitz system.

Theorem 7 in conjunction with Theorem 8 may be used to prove existence theorems for differential or integral equations (Hyers [1, $2])$. By interpreting the space $L$ in different ways a variety of existence theorems can be proved. For instance, on taking $L$ to be the space $(s)$ of all real sequences $x=\left(x_{1}, x_{2}, \cdots\right)$, topologized by component-wise convergence, we can obtain an existence theorem for an infinite system of ordinary differential equations. Again if $L$ is taken to be the weakly topologized Hilbert space $\mathfrak{K}$, one may establish an existence theorem for "weak" differential equations for functions whose values lie in Hilbert space.

4. Pseudo-norms. The notion of a pseudo-norm, introduced by J. von Neumann [1] for the case where the 1.t.s. is locally convex, is both useful and suggestive in theories of linear transformations, linear functionals, differentials, and so on. It springs from the same idea already used in proving Kolmogoroff's normability theorem, and in connection with locally bounded spaces, namely, the Minkowski functional. It differs from a norm (or the quasi-norm introduced in \$2) in that the vanishing of the pseudo-norm of an element $x$ does not imply that $x=\theta$. The following treatment is based upon the author's generalization ${ }^{18}$ of von Neumann's pseudo-norm, so that it may be applied to any 1.t.s.

Definition 4.1. An arbitrary set $D$ with elements $a, b, c, \cdots$ together with a binary relation $>$ is called a directed system ${ }^{19}$ if the following postulates are satisfied:

(i) either $a>b$ or $a \ngtr b$ for every pair $a, b$ in $D$;

(ii) if $a>b$ and $b>c$ then $a>c$;

(iii) given $a \in D$ and $b \in D$ there exists $c \in D$ such that $c>a$ and $c>b$.

Properties (i) and (ii) state that $D$ is partially ordered, and (iii) is the "composition" property of Moore and Smith.

Definition 4.2. A linear space $L$ will be said to be pseudo-normed

${ }^{18}$ Hyers [5]. See also LaSalle [2].

${ }^{19}$ Moore and Smith [1]; G. Birkhoff [2]; Tukey [1]. In our notation $a>b$ includes the statement $a=b$. 
with respect to a directed system $D$ if there exists a real-valued functional $|x|_{d}$ defined for all $x \in L$ and $d \in D$ which satisfies the following postulates.

(1) $|x|_{d} \geqq 0 ;|x|_{d}=0$ for all $d \in D$ implies $x=\theta$.

(2) $\left.\alpha x\right|_{d}=|\alpha| \cdot|x|_{d}$ for all real $\alpha$ and every $x \in L$.

(3) Given $e \in D$ there exists $d \in D$ such that $|x+y|_{e} \leqq|x|_{d}+|y|_{d}$ for all $x$ and $y$ in $L$.

(4) $|x|_{d} \geqq|x|_{\text {e whenever }} d>e$, and for all $x$ in $L$.

RemarK. The property (3) in Definition 4.2 is due to LaSalle [2]. A weaker property was used in the author's original definition.

As a simple example of a pseudo-norm, consider the space $(s)$ of all real sequences $x=\left(x_{1}, x_{2}, \cdots\right)$ and take $D$ to be the ordered set of positive integers. Then the functional $|x|_{d}=\max \left\{\left|x_{i}\right| ; 1 \leqq i \leqq d\right\}$ is a pseudo-norm for $(s)$ with respect to the set of positive integers.

Having defined a pseudo-norm for a space, the topology, obtained by defining $x$ to be a limit point of a set $S$. if, given $\delta>0$ and $d \in D$, there exists a point $y \neq x$ such that $|x-y|_{d}<\delta$, is called the topology generated by the pseudo-norm. In the example just given the pseudonorm generates a topology equivalent to the usual metric topology for $(s)$.

THEOREM 9. Every pseudo-normed linear space $L$ is a l.t.s. in which the pseudo-norm generates the topology of L. Conversely, given any l.t.s. $L$, there exists a directed system $D$ with respect to which $L$ may be pseudonormed in such a way that the pseudo-norm generates the topology of $L .{ }^{20}$

Corollary. The directed system $D$ for a l.t.s. may be identified with a certain complete neighborhood system $\{U\}$ of the origin, in which $U_{1}>U_{2}$ means $U_{1} \subset U_{2}$.

The directed system $D$ for a 1.t.s. $L$ will be called $a$ directed system associated with $L$.

Theorem 9 affords an alternative definition of a 1.t.s. In some cases it seems simpler to define a pseudo-norm directly instead of defining an equivalent neighborhood topology.

A pseudo-norm is called triangular if it satisfies the postulate:

(3a) $|x+y|_{d} \leqq|x|_{d}+|y|_{d}$ for all $x, y \in L$ and $d \in D$; that is, if $d=e$ in property (3) of Definition 4.2.

The "pseudo-metric" of von Neumann [1] is a triangular pseudonorm for which $D$ is a suitably chosen complete neighborhood system of $\theta$. It is possible to define a triangular pseudo-norm for a l.t.s. $L$ if and only if $L$ is locally convex. ${ }^{21} \mathrm{~A}$ good many properties of 1.t.s. and

${ }^{20}$ Hyers [5]; LaSalle [2].

21 von Neumann [1]. 
their subsets can be stated conveniently in terms of the pseudo-norm. Thus, it is easily shown on the basis of Theorem 9 that a subset $S$ of a 1.t.s. $L$ is bounded if and only if, given $d \in D$, there exists $\mu>0$ such that $|x|_{d}<\mu$ for all $x \in S$.

The notion of pseudo-norm has been generalized still more by J. P. LaSalle $[1,3]$ to apply to linear spaces in which the "scalars" are elements of a normed ring instead of real numbers, and also to topological spaces which need not be linear spaces.

5. Linear transformation and functionals. In this section we shall consider additive transformations $T(x)$ on a l.t.s. $L_{1}$ to a l.t.s. $L_{2}$. The following theorem was proved by Wehausen [1] for the case in which $L_{1}$ and $L_{2}$ are both locally convex. However, the proof remains unaltered for the present more general statement.

Theorem 10. Let $L_{1}$ and $L_{2}$ be two l.t.s. and let $D_{1}$ and $D_{2}$ be associated directed systems for $L_{1}$ and $L_{2}$, respectively. An additive transformation $T$ on $L_{1}$ to $L_{2}$ is continuous if and only if to each $e$ in $D_{2}$ there corresponds a d in $D_{1}$ and a real number $\mu \geqq 0$ such that $|T(x)|_{e} \leqq \mu|x|_{d}$, for all $x$ in $L_{1}$.

Corollary. A linear continuous transformation on $L_{1}$ to $L_{2}$ takes bounded sets into bounded sets.

In the study of linear operators on $L_{1}$ to $L_{2}$, one of the natural questions which arises is that of finding a suitable topology for the set $\tau$ of all continuous linear transformations $T$ on $L_{1}$ to $L_{2}$ which will make $\mathcal{T}$ a linear topological space. A satisfactory topology was given by LaSalle [2] in terms of a pseudo-norm. The focus of attention is the set $B_{1}$ of all bounded sets $B$ of $L_{1}$ which contain the origin, rather than a neighborhood system of the origin in $L_{1} . B_{1}$ is a directed system if we define $B>B^{\prime}$ to mean $B C B^{\prime}$. Consider the set $D$ of all pairs $e=(d, B)$, where $d \in D_{2}, B \in \mathcal{B}_{1}$, and $D_{2}$ is a directed system associated with $L_{2}$. Then $E$ is also a directed system, if we define $e>e^{\prime}$ to mean that $d>d^{\prime}$ and $B>B^{\prime}$.

THEOREM 11. For each $T \in \mathcal{T}$ and each $e \in E$, put $|T|_{0}=$ sup $\left\{|T(x)|_{d} ; x \in B\right\}$. Then $|T|_{0}$ is a pseudo-norm for the linear space $\mathcal{T}$, and $E$ is an associated directed system. In case $L_{2}$ is locally convex, then $|T|_{0}$ is a triangular pseudo-norm. ${ }^{22}$

In particular, Theorem 11 provides a topology for the space $L^{*}$ of all linear functionals on a 1.t.s. $L$. An equivalent topology in this special case was used by Bourgin [1].

* LaSalle [2, Theorems 5, 6]. 
We now turn to the special case of functionals on $L$. The following existence theorems, due to Wehausen [1] and LaSalle [2] respectively, may be proved on the basis of the Hahn-Banach theorem (Banach [1, p. 27, Theorem 1]). These two theorems once again emphasize the importance of convexity properties in connection with linear functionals.

THEOREM 12. If $L$ is a locally convex l.t.s. and $D$ is an associated directed system, then for any $x_{0} \in L$ and any $d \in D$ there exists a linear continuous functional $f(x)$ defined on $L$ with the property that $f\left(x_{0}\right)$ $=\left|x_{0}\right| d$.

It is easily shown by example (for example, the sequence space $l^{1 / 2}$ ) that this condition of local convexity, while sufficient, is not necessary for the existence of non-null linear continuous functionals. This remark adds interest to LaSalle's result:

THEOREM 13. A necessary and sufficient condition that there exist a non-null continuous linear functional on a l.t.s. $L$ is that there exist a convex open set which is properly contained in $L$.

The question of the existence of linear continuous functionals in function spaces of the type $L^{p}$ and $l^{p}$, where $0<p<1$, has been studied by M. M. Day [1]. In particular it was shown that every continuous linear functional defined on the space $L^{p}$ of real functions $f(x)$ on the interval $(0,1)$ is identically zero when $0<p<1$.

In the light of Theorems 12 and 13 the desirability of requiring local convexity in order to establish a satisfactory theory of linear functionals on a 1.t.s. becomes apparent. In the case of a locally convex 1.t.s., a good many generalizations of theorems on linear functionals which are well known for Banach spaces can be carried through. In particular, theorems of the Helly type (Banach [1, pp. 55-57]) on the extension of functionals, which are important for their applications to the problem of moments and the solution of infinite systems of linear equations, have been generalized by Wehausen [1] and Bourgin [1].

The relationship between a locally convex 1.t.s. $L$ and the space $L^{*}$ of linear functionals on $L$ has been studied from a new viewpoint by G. W. Mackey $[1,2]$. He starts with an abstract linear space $X$, singles out a certain subset of the set of all linear (additive and homogeneous) functionals on $X$, and considers the problem of what topologies in $X$ will make the distinguished set of functionals the continuous functionals. In the case of normed spaces, there is a theorem of Fichtenholz [1] to the effect that two norms in the same linear space give 
the same topology if and only if the same linear functionals are continuous with respect to both norms. Such is not the case, however, in the case of locally convex l.t.s., as shown by the example of a Banach space with its weak and strong topologies. This leads to the concepts of relatively weak and relatively strong 1.t.s., and to several theorems involving these notions. (In the example just referred to, the norm topology $t_{1}$ is relatively strong, and the weak topology $t_{2}$ obtained from the set of all linear continuous functionals is relatively weak. Any other locally convex topology for the same space and leading to the same set of continuous linear functionals will be stronger than $t_{2}$ and weaker than $t_{1}$.) In particular it is shown that a locally convex 1.t.s. of the second category is relatively strong. This theorem generalizes Wehausen's result [1] that an infinitely-dimensional Banach space with its weak topology is of the first category.

6. Differentials. In functional analysis a number of definitions of a differential have been used. ${ }^{28}$ Perhaps the simplest is the "weak" or Gateaux differential $d f(x+\alpha \delta x) /\left.d \alpha\right|_{\alpha=0}$ which is simply the "variation" used in the calculus of variations. However, the Gateaux differential does not have many of the usual properties associated with the total differential for functions of two or more real variables, and we shall be interested in the generalization of the total differential. At this point it might be well to list the properties which a differentiable function should have. Denoting the differential of $f(x)$ with increment $\delta x$ by $d f(x ; \delta x)$ we shall require that (I) if a function is differentiable at a point it is continuous at this point; (II) the composite function rule holds, that is, if $g(x)=f(\phi(x))$ and $d \phi$ and $d f$ exist then $d g$ exists and $d g(x ; \delta x)=d f(y ; d y)$, where $y=\phi(x), d y=d \phi(x ; \delta x)$; (III) the differential is linear and continuous in the increment; (IV) $d f(x ; \delta x)$ is a first order approximation to the difference $f(x+\delta x)-f(x)$ when $\delta x$ is "close" to $\theta$.

Property (IV) is purposely left vague. The various definitions which will be discussed differ principally in the exact interpretation of this property.

The standard definition of a differential of a function on $E_{1}$ to $E_{2}$, where $E_{1}$ and $E_{2}$ are linear normed spaces, is due to Fréchet [1]. A function on $E_{1}$ to $E_{2}$ is Fréchet differentiable at the point $x$ with the differential $d f(x ; \delta x)$ if $d f(x ; \delta x)$ is a linear continuous function of $\delta x$ on $E_{1}$ to $E_{2}$ such that $\lim _{h \rightarrow \theta}\|f(x+h)-f(x)-d f(x ; h)\| /\|h\|=0$. It is well known that the Fréchet differential has the four properties listed above. The definition shows the sense in which it satisfies (IV).

${ }^{23}$ For example, see Fréchet [1, 2]. For a discussion see Graves [1]. 
The differential calculus has been extended to include functions whose arguments and values lie in 1.t.s. and also in topological groups by A. D. Michal and his students. The first step in this direction was made by Michal and Paxson $[1,2]$ in 1936. However, their definition did not prove entirely satisfactory, since the domain and range of the functon had to lie in the same space, and the rule (II) for the differentiation of composite functions was not demonstrated. Since then a number of different definitions have been proposed having the properties (I), (II), (III), (IV). We consider first Michal's $M$-differential. ${ }^{24}$

Definition 6.1. Let $L_{1}, L_{2}$ be 1.t.s., and let $f(x)$ be defined on a neighborhood $N$ of a point $x_{0} \in L_{1}$ to $L_{2}$. The function $f(x)$ will be said to be $M$-differentiable at $x=x_{0}$, and $d f\left(x_{0} ; \delta x\right)$ will be called an $M$-differential of $f(x)$ at $x=x_{0}$ with increment $\delta x$ if and only if

(1) $d f\left(x_{0} ; \delta x\right)$ is a linear continuous function on $L_{1}$ to $L_{2}$;

(2) there exists a function $\epsilon\left(x_{0}, x_{1}, x_{2}\right)$ with arguments in $L_{1}$ and values in $L_{2}$ such that

(a) $\epsilon\left(x_{0}, \theta, x\right)=\theta$ for all $x \in L_{1}$;

(b) $\epsilon\left(x_{0}, x_{1}, \lambda x_{2}\right)=\lambda \epsilon\left(x_{0}, x_{1}, x_{2}\right)$; for all $\lambda>0$, for all $x_{1}$ in some neighborhood of $\theta \in L_{1}$ and for all $x_{2} \in L_{1}$;

(3) there exists a neighborhood $U$ of $\theta \in L_{1}$ such that $f\left(x_{0}+\delta x\right)$ $-f\left(x_{0}\right)-d f\left(x_{0} ; \delta x\right)=\epsilon\left(x_{0}, \delta x, \delta x\right)$ for all $\delta x \in U$.

The $M$-differential can be shown to have the properties I, II, III, and the Definition 6.1 shows the sense in which it satisfies IV. Every $M$-differential is a Gateaux differential, so that the function $d f\left(x_{0} ; \delta x\right)$ is unique, for a given $x_{0}$ and a given $f(x)$. When $L_{1}$ and $L_{2}$ are normable spaces, every Fréchet differential is also an $M$-differential, but the converse statement is not true, as is shown by the following example.

Let $L_{1}$ be the linear normed space of all sequences $x=\left(x_{1}, x_{2}, \cdots\right)$ such that only a finite number of the $x_{i}$ 's are different from zero, where $\|x\|=\sup \left\{\left|x_{i}\right| ; 0<i<\infty\right\}$. Let $L_{2}$ be the linear normed space of all bounded sequences, with the norm defined in the same way. Define the function $f(x)=\left(f_{1}(x), f_{2}(x), \cdots\right)$ on $L_{1}$ to $L_{2}$ by means of the formula $f_{r}(x)=\left(r^{1 / 2}+r^{3 / 2} x_{r}^{2}\right)^{-1}, r=1,2,3, \cdots$. It can be shown that $f(x)$ is $M^{\prime}$-differentiable at the origin with $d f(\theta ; \delta x)=\theta$, by defining $\epsilon(\theta, y, z)=-r^{1 / 2} y_{r} z_{r} /\left(1+r y_{r}^{2}\right)$ and verifying that the conditions (1), (2), (3) of Definition 6.1 are satisfied.

Now if we assume that $f(x)$ is Fréchet differentiable, then there is a $\delta>0$ such that $\|\epsilon(\theta, y, y)\| \leqq\|y\| / 4$ for $\|y\|<\delta$. Choose $n$ so large that $n^{1 / 2}>\delta^{-1}$, and choose $y$ so that $y_{r}=0$ for $r \neq n$, and $y_{n}=n^{-1 / 2}$. Clearly

4 Michal $[1,2]$. A number of other definitions of differentials have been given by the same author. See Michal [1-5]. 
$\|y\|<\delta$. However, $\|\epsilon(\theta, y, y)\| /\|y\|=1 / 2$. Hence $f(x)$ is not Fréchet differentiable at the origin. This example shows that the $M$-differential satisfies the condition (IV) in an essentially weaker sense than does the Fréchet differential.

Since Fréchet's original definition leans so heavily on the concept of a norm, it is natural to try to phrase a definition in terms of pseudonorms (Hyers [6]).

Definition 6.2. Let $L_{1}$ and $L_{2}$ be 1.t.s., and let $D_{1}, D_{2}$ be associated directed systems. The function $f(x)$ on an open set $G \subset L_{1}$ to $L_{2}$ will be said to be $F$-differentiable at the point $x$ of $G$, and $d f(x ; \delta x)$ will denote its $F$-differential if

(a) $d f(x ; h)$ is linear and continuous in $h$ on $L_{1}$ to $L_{2}$;

(b) for every $e \in D_{2}$ there corresponds $d \in D_{1}$ such that, given $\eta>0$, there exists $\delta>0$ with $|f(x+h)-f(x)-d f(x ; h)|_{e} \leqq \eta|h|_{d}$ whenever $|h|_{d}<\delta$.

Note that $d$ depends only on $e$, while $\delta$ depends on $e, d$ and $\eta$. It can be shown that every $F$-differentiable function is also $M$-differentiable. The $F$-differential has the properties I-IV. In addition, when $L_{1}$ and $L_{2}$ are normable, the $F$-differential reduces to the Fréchet differential. It follows that the $M$-differential is more general than the $F$-differential.

The notion of a differential for functions whose arguments and values are in certain abelian topological groups was also introduced by Michal $[3,4]$. The definition of the differential was of the same general character as Definition 6.1, except that $\lambda$ was, of course, restricted to be an integer, and a stronger uniformity condition was imposed on the $\epsilon$-function. This uniformity property was strong enough to make the definition reduce to that of Fréchet when the topological abelian groups were taken to be linear normed spaces and when the homogeneity property of the $\epsilon$-function was assumed to hold for all real scalars. However, for 1.t.s. this modified $M$-differential is less general than the $F$-differential. Higher order differentials and their properties were investigated by the same author. ${ }^{25}$

Later LaSalle (1) introduced differentials for transformations of a still more general type of space-a vector space in which the scalar multipliers were replaced by elements of a valued ring. His definition makes use of a suitably generalized pseudo-norm. Differentials for functions on noncommutative topological groups have been studied by $\mathrm{K}$. Millsaps [1].

E. J. Pinney [1] has studied the calculus of variations for functionals on a 1.t.s., making use of the $m$-differential.

${ }^{25}$ Michal [5]. 
Although a good many definitions of differentials have been given and many of their more elementary properties have been studied, much remains to be done in the development of the differential calculus for transformations of one 1.t.s. into another. In particular it would be interesting to obtain a generalization of the implicit function theorems proved by Hildebrandt and Graves [1] for the case of linear normed spaces. These theorems have many applications in functional analysis and it is desirable to extend their range of application still farther.

7. Convergence and completeness. We have seen how the topology of a 1.t.s. could be generated by means of neighborhoods or by means of pseudo-norms. Another equivalent way is to use a suitably generalized notion of convergence. In non-metric topologies it is clearly not sufficient to consider merely the convergence of a sequence in the ordinary sense, since, by von Neumann's example already referred to, a point $p$ may be a limit point of a set and yet no sequence of elements of the set may converge to the point. However, by using some ideas of Moore and Smith [1] on the convergence of integrals, Garrett Birkhoff [2] succeeded in generalizing the notion of convergence so that the convergence topology is equivalent to the neighborhood topology, even in very general spaces. ${ }^{26}$ Given an abstract directed system $D$ (Definition 4.1) and a topological space $T$, a directed $\operatorname{set}^{27} x_{\alpha}$ is defined to be a function on $D$ to $T$. A directed set $x_{\alpha}$ is said to converge to the limit point $x$ if, for every neighborhood $V_{x}$ of $x$, there exists $\alpha_{0} \in D$ such that $\alpha>\alpha_{0}$ implies that $x_{\alpha} \in V_{x}$. Thus the generalization consists in replacing the ordered set of positive integers coming into the definition of a sequence by directed systems $D$.

In terms of this concept of convergence Birkhoff defined "completeness" of a 1.t.s. as follows. First, a directed set $x_{\alpha}$ will be called fundamental if, for every neighborhood $U$ of the origin, there exists $\alpha_{0}$ such that $\alpha>\alpha_{0}, \beta>\alpha_{0}$ imply that $x_{\alpha}-x_{\beta} \in U$. A 1.t.s. $L$ will be said to be complete if every fundamental directed set converges to some $x \in L$. Completeness is an extensionally obtainable property, ${ }^{28}$ although most of the "natural" instances of 1.t.s. are not complete. For example, weak Hilbert space is not complete, in Birkhoff's sense.

Several other types of completeness have been defined for 1.t.s., all of which coincide in metric spaces. The most obvious definition is that of "sequential completeness" already used in connection with the

${ }^{26}$ For a discussion see Tukey [1].

${ }^{27}$ The term "directed function" might have been better.

${ }^{28}$ Birkhoff [2]. 
fixed point theorem of a previous section. This is the weakest type of completeness.

J. von Neumann [1] has defined "topological completeness" by generalizing another criterion for completeness in metric spaces. A subset $S$ of a 1.t.s. is called totally bounded if for every neighborhood $U$ of the origin there is a finite set $x_{1}, x_{2}, \cdots, x_{n}$ of points of $L$ such that the family of sets $x_{i}+U, i=1, \cdots, n$, covers $S$. Then $L$ is said to be topologically complete if every totally bounded set is compact. It can be shown ${ }^{29}$ that every topologically complete 1.t.s. is also sequentially complete. In particular weak Hilbert space $\mathfrak{T C}$ is topologically and hence sequentially complete.

Still other definitions of completeness have been given by Šmulian [1] and Taylor $[1,2]$. Smulian calls a 1.t.s. quasi-complete if every totally bounded closed set is complete in Birkhoff's sense, and Taylor calls a 1.t.s. boundedly complete if every bounded fundamental directed set is convergent, that is, if every bounded closed set is complete.

If we denote by $C$ the class of complete 1.t.s., by $B$ the class of those which are boundedly complete, by $Q$ those which are quasi-complete, by $T$ the topologically complete and by $S$ the sequentially complete 1.t.s., then we have the following inclusion relations:

$$
C \subset B \subset Q \subset T \subset S \text {. }
$$

An important theorem on metric spaces states that every complete metric space is of the second category. However, this is not true ${ }^{30}$ for all 1.t.s. belonging to the classes $S, T, Q$ or $B$.

The notions of convergence of directed sets and the various notions of completeness and compactness arising therefrom have been applied to the study of Banach spaces by a large and still growing number of authors, including Alaoglu, Bourgin, Day, Dunford, Goldstine, Milman, Pettis, Smulian, Taylor and several others. The ideas centering around the notion of weak (sequential) convergence as developed by Banach [1] are generalized by the use of the convergence of directed sets instead of sequences. This often makes possible the elimination of the hypothesis of separability which usually occurs in Banach's results. To quote only one example, a well known theorem ${ }^{31}$ states that if a Banach space $E$ is separable and has a weakly compact unit sphere then $E$ is reflexive. An equivalent statement of this result may be obtained ${ }^{32}$ by replacing the weak compactness condition by the condition that $\varepsilon$ be topologically complete. Goldstine [1] generalized this result by the use of convergence of directed sets, and obtained a

\footnotetext{
${ }^{29}$ von Neumann [1].

${ }^{30}$ Wehausen [1]; Mackey [2].

31 Banach [1, p. 189].

32 Taylor $[2, \S 6]$.
} 
necessary and sufficient condition for reflexivity. Goldstine's result may be stated in either of the two forms:

A Banach space $E$ is reflexive if and only if

(a) $\varepsilon$ is boundedly complete, ${ }^{33}$

(b) $\varepsilon$ is quasi-complete. ${ }^{34}$

Several other conditions for reflexivity have been given by Milman, Day, Bourgin and others in terms of various types of weak compactness of the unit sphere. However, for lack of space we are unable to pursue this interesting topic further.

8. Conclusion. There are several topics which we must leave with but the briefest mention. The subject of integration of functions whose values are in 1.t.s. has been considered by a number of authors. In particular, integrals of the Lebesgue type have been defined by G. Birkhoff [2], R. S. Phillips [1], and the present author. ${ }^{85}$ The work has been carried the farthest by Phillips, who showed that by properly choosing the neighborhood topology for the special case of a Banach space, his integral can be made to include the integrals of Birkhoff, Dunford, Gelfand, and Pettis, depending on the "strength" of the topology used. This is an example of how the concept of 1.t.s. may be used to unify various theories which appear quite diverse from the standpoint of Banach space theory.

The theory of almost periodic functions has been studied by S. Bochner and J. von Neumann [1] in a very general setting, in which the arguments of the functions lie in groups, and the values in a 1.t.s.

Another interesting topic is the relation between 1.t.s. and abelian topological groups. The question of characterizing the 1.t.s. among all abelian topological groups has been answered by E. W. Paxson [4] and R. C. James [1]. The question of characterizing the subgroups if a 1.t.s. has not yet been satisfactorily solved in general. Partial answers have been given by James [2] and Hyers [5].

In conclusion we mention another unsolved problem, namely, under what conditions does a linear transformation of one 1.t.s. into another have an inverse? The general type of existence proof used for metric spaces seems unavailable here, since non-metric 1.t.s. are usually of the first category, even when they satisfy completeness conditions. Apparently little has been done on this problem even for special types of transformations such as $T(x)=x+G(x)$ where $G(x)$ is completely continuous.

${ }^{33}$ Taylor $[1,2]$. $\varepsilon$, as usual, denotes the Banach space with its weak topology.

34 Smulian [1].

${ }^{35}$ Hyers [1]. 


\section{BIBLIOGRAPHY}

L. AlaOglu

1. Weak topologies of normed linear spaces, Ann. of Math. vol. 41 (1940) pp. 252267.

P. AleXANDRoff AND H. Hopf

1. Topologie, vol. I, Berlin, 1935.

S. BANACH

1. Operations lineaires, Warsaw, 1932.

\section{GARRETT BIRKHOFF}

1. A note on topological groups, Compositio Math. vol. 3 (1936) pp. 427-430.

2. Moore-Smith convergence in general topology, Ann. of Math. vol. 38 (1937) pp. 39-56.

G. D. BiRKhoff AND O. KellogG'

1. Invariant points in function space, Trans. Amer. Math. Soc. vol. 23 (1922) pp. 96-115.

S. BochNeR AND J. Von NeUMANN

1. Almost periodic functions on groups. II, Trans. Amer. Math. Soc. vol. 37 (1935) pp. 21-50.

D. G. BOURGIN

1. Linear topological spaces, Amer. J. Math. vol. 65 (1943) pp. 637-659.

2. Some properties of Banach spaces, Amer. J. Math. vol. 64 (1942) pp. 597-612. M. M. DAX

1. The spaces $L^{p}$ with $0<p<1$, Bull. Amer. Math. Soc. vol. 46 (1940) pp. 816-823.

2. A property of Banach spaces, Duke Math. J. vol. 8 (1941) pp. 763-770.

M. EIDELHEIT AND S. MAZUR

1. Eine Bemerkung uber die Raume von Typus $(F)$, Studia Mathematica vol. 7 (1938) pp. 159-161.

G. FICHTENHOLZ

1. Sur les fonctionelles linéaires, continues au sens gênéralisé, Rec. Math. (Math. Sbornik) N.S. vol. 4 (1938) pp. 192-213.

M. FRECHET

1. La differentielle dans l'analyse générale, Ann. Ecole Norm. vol. 42 (1925) pp. 293-323.

2. Les espaces topologiquement affines, Acta Math. vol. 47 (1926) pp. 25-52.

3. Les espaces abstraits, Paris, 1928.

H. GoLDSTINE

1. Weakly complete Banach spaces, Duke Math. J. vol. 4 (1938) pp. 125-132.

L. M. GRAVES

1. Topics in the functional calculus, Bull. Amer. Math. Soc. vol. 41 (1935) pp. 641-662.

2. On the completing of a Hausdorff space, Ann. of Math. vol. 38 (1937) pp. 61-64.

T. H. Hildebrandt aNd L. M. Graves

1. Implicit functions and their differentials in general analysis, Trans. Amer. Math. Soc. vol. 29 (1927) pp. 127-153.

D. H. HYERS

1. Integrals and functional equations in linear topological spaces, California Institute of Technology thesis, 1937.

2. On functional equations in linear topological spaces, Proc. Nat. Acad. Sci. U.S.A. vol. 23 (1937) pp. 496-499.

3. A note on linear topological spaces, Bull. Amer. Math. Soc. vol. 44 (1938) pp. 76-80. 
4. Locally bounded linear topological spaces, Revista de Ciencias vol. 41 (1939) pp. 555-574.

5. Pseudo-normed linear spaces and Abelian groups, Duke Math. J. vol. 5 (1939) pp. 628-634.

6. A generalization of Fréchet's differential, Proc. Nat. Acad. Sci. U.S.A. vol. 27 (1941) pp. 315-316.

R. C. JAMES

1. Linearly arc-wise connected topological Abelian groups, Ann. of Math. vol. 44 (1943) pp. 93-102.

2. Topological groups as subgroups of linear topological spaces, Duke Math. J. vol. 10 (1943) pp. 441-453.

A. KolmogorofF

1. Zur Normierbarkeit eines topologischen linearen Raumes, Studia Mathematica vol. 5 (1934) pp. 29-33.

J. P. LASALlE

1. Pseudo-normed linear sets over valued rings, California Institute of Technology Thesis, 1941.

2. Pseudo-normed linear spaces, Duke Math. J. vol. 8 (1941) pp. 131-136.

3. Topology based upon the concept of a pseudo-norm, Proc. Nat. Acad. Sci. U.S.A. vol. 27 (1941) pp. 448-457.

G. W. MACKEY

1. On infinite dimensional linear spaces, Proc. Nat. Acad. Sci. U.S.A. vol. 29 (1943) pp. 216-221.

2. On convex topological linear spaces, Proc. Nat. Acad. Sci. U.S.A. vol. 29 (1943) pp. 315-319.

S. MAzUR AND W. ORLICZ

1. Über Folgen linearen Operationen, Studia Mathematica vol. 4 (1933) pp. 152 157.

A. D. Michal

1. Differential calculus in linear topological spaces, Proc. Nat. Acad. Sci. U.S.A. vol. 24 (1938) pp. 340-342.

2. General differential geometries and related topics, Bull. Amer. Math. Soc. vol. 45 (1939) pp. 529-563.

3. Differentials of functions with arguments and values in topological abelian groups, Proc. Nat. Acad. Sci. U.S.A. vol. 26 (1940) pp. 356-359.

4. First order differentials of functions with arguments and values in topological abelian groups, Revista de Ciencias (in press).

5. Higher order differentials of functions with arguments and values in topological abelian groups, Revista de Ciencias vol. 42 (1940) pp. 155-177.

A. D. Michal and E. W. Paxson

1. La differentielle dans les espaces abstraits linéaires avec une topologie, C. R. Acad. Sci. Paris vol. 202 (1936) pp. 1741-1743.

2. The differential in abstract linear spaces with a topology, Comptes Rendus des Séances de la Société des Sciences et des Lettres de Varsovie vol. 29 (1936) pp. 106-121.

K. Millsaps

1. Differential calculus in topological groups, Revista de Ciencias vol. 44 (1942) pp. 485-492.

2. Differential calculus in topological groups II, Revista de Ciencias vol. 45 (1943) pp. 45-52. 
E. H. Moore and H. L. Smith

1. A general theory of limits, Amer. J. Math. vol. 44 (1942) pp. 102-121.

E. W. PAxson

1. Analysis in linear topological spaces, California Institute of Technology Thesis, 1937.

2. Sur un espace fonctionnel abstrait, Revista de Ciencias vol. 42 (1940) pp. 817822.

3. Les équations differentielles dans les espaces linéaires et topologiques, Revista de Ciencias vol. 42 (1940) pp. 823-826.

4. Linear topological groups, Ann. of Math. vol. 40 (1938) pp. 575-580.

R. S. PHILlips

1. Integration in a convex linear topological space, Trans. Amer. Math. Soc. vol. 47 (1940) pp. 114-145.

E. J. Pinney

1. The calculus of variations in abstract spaces and related topics, California Institute of Technology Thesis, 1942.

L. PontrJagin

1. Topological groups, Princeton, 1939.

F. RIESZ

1. Über lineare Funktionalgleichungen, Acta Math. vol. 41 (1918) pp. 71-98.

J. SCHAUDER

1. Der Fixpunktsatz in Funktionalraumen, Studia Mathematica vol. 2, pp. 171180.

V. SMULIAN

1. Linear topological spaces and their connection with Banach spaces, C. R. (Doklady) Acad. Sci. URSS. vol. 22 (1939) pp. 471-473.

A. E. TAYLOR

1. The weak topologies of Banach spaces, Proc. Nat. Acad. Sci. U.S.A. vol. 25 (1939) pp. 438-440.

2. The weak topologies of Banach spaces, Revista de Ciencias vol. 43 (1941) pp. 355-

J. TUKEY 365, 465-474, 667-674 and vol. 44 (1942) pp. 45-63.

1. Convergence and uniformity in topology, Princeton, 1940.

J. von NEUManN

1. On complete topological spaces, Trans. Amer. Math. vol. 37 (1935) pp. 1-20.

2. Zur Algebra der Funktionaloperatoren und theorie der normalen Operatoren, Math. Ann. vol. 102 (1929) pp. 370-427.

A. Tychonofr

1. Ein Fixpunktsatz, Math. Ann. vol. 111 (1935) pp. 767-776.

J. V. WeHAUSEN

1. Transformations in linear topological spaces, Duke Math. J. vol. 4 (1938) pp. 157-169.

A. WEIL

1. Sur les espaces a structure uniforme, Actualités Scientifiques et Industrielles, No. 551, Paris, 1938.

H. WEYL

1. On the differential equation of the simplest boundary layer problems, Ann. of Math. vol. 43 (1942) pp. 381-407.

The University of Southern California and

The California Institute of Technology 